\title{
Les conséquences du vrai sens de IAגCTHPION.
}

Par C. Bruston, Montauban.

On doit être très reconnaissant à M. Deissinann d'avoir pris la peine

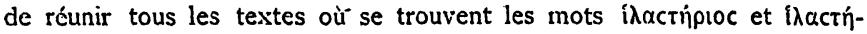

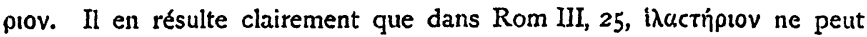
guère ĉtre un adjectif, comme on l'a cru quelquefois, mais doit être un substantif, et que ce substantif ne signifie pas (en tout cas, pas nécessairement ni même habituellement) une victime propitiatoire, mais plutôt une offrande $(\alpha \dot{\alpha} v \dot{\theta} \theta \eta \alpha)$ propitiatoire ou expiatoire, offerte à la Divinité.

S'il en est ainsi, cette offrande (la personne de Jésus) fut offerte par Jésus lui-même à Dieu. De sorte que, sans ètre exprimée explicite-

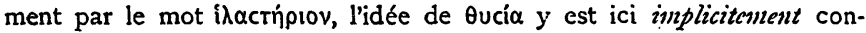
tenue, - ce qui résulte aussi du contexte, surtout des mots év $\tau \hat{\varphi}$

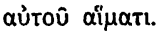

Du sens de i $\lambda \alpha$ crńpiov ainsi déterminé résultent plusieurs conséquences importantes, que $M$. Deissmann semble n'avoir pas entrevues, mais qu'il me parait difficile de n'en pas tirer logiquement.

1. D'abord, puisqu'un îacríprov est une offrande faite à Dicu par des hommes, il est impossible que Paul ait voulu dire que Dieu avait exposé Jésus-Christ aux hommes comme îactńpıov. Une telle idée serait

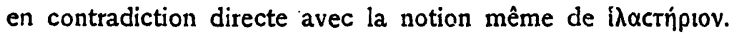

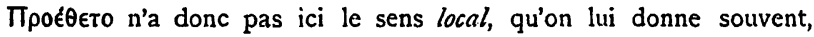
adopté par M. Deissmann lui-même, mais le sens temporel, confirmé d'ailleurs par l'usage qu'en fait l'apôtre (Eph I, 9; Rom I, I3). C'est en

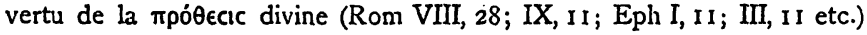
que J.-C. a été plus tard i $\lambda \alpha c$ ńpiov. Tant de textes parallèles ne permettent pas d'en douter.

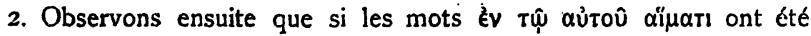
rattachés à ce qui les précéde immédiatement, c'est vraisemblablement parce qu'on supposait que ìactńpiov renfermait l'idée d'une victime 
immoléc, sanglante. P'uisqu'il n'en est rien, il n'y a aucune raison de considerer ces 4 mots comme faisant partie du membre de phrase relatif ov $\pi \rho \circ \in \theta \in \tau o k \tau \lambda$. Il est beaucoup plus naturel, au contraire, d'en fairc la

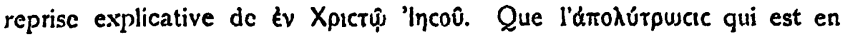
$\mathcal{F}$-C. soit aussi, spécialement, en son sang, c'est là une idte très fréquente, $^{x}$ tandis que l'idce que J.-C. est îactíprov en son sang, dejà assez forcée, obscurc et invraisemblable en elle-même, le parait d'autant plus maintenant que nous savons qu'un ìactípıov n'etait pas un sacrifice sanglant.

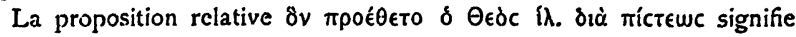
donc simplement que Dieu avait jadis (de toute éternité) décidé que J.-C. serait un jour un $\{\lambda$. par le moyen de la foi. En fait, il a été aussi une victime sanglante; mais cette idée exprimée dans la phrase principale, ne l'est pas dans la proposition relative.

3. Il résulte de cette construction que toute la suite de la phrase:

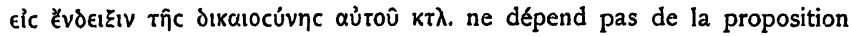
relative $\delta v \pi \rho \in \in \in \epsilon \in$ to $\delta \theta \in \dot{c} c \kappa \tau \lambda$., qui serait alors démesurément longue

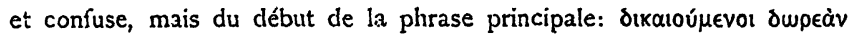

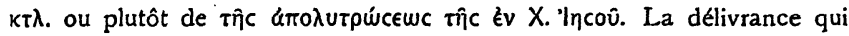
est en J.-C., dans son sang à liui (remarquez la place du pronom), et non dans celui des animaux, comme sous l'ancienne alliance, eut pour but, dans la pensće de Dieu, „la manifestation de sa justice" (eic èvঠeızı

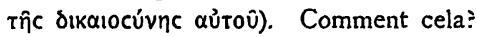

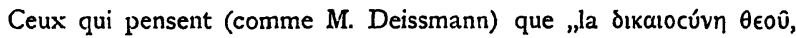
dans les 4 passages où elle est mentionnée (au début de l'épitre) n'est pas un attribut de Dieu, mais la justice accordée par Dieu au croyant, par grâce et en Christ" auräient vraisemblablement quelque peine à l'expliquer. Car de ce que Dieu délivre (ảmodúrpwac) les hommes de la condamnation et de la mort éternelle, il n'en résulte pas nécessairement qu'il veuille leur communiquer sa justice propre; et une telle communication de la justice divine pourrait difficilement être appelée "la manifestation de la justice de Dieu." Pour être manifestée, en effet, il faut qu'une chose existe et qu'elle ait été cachiee au moins pendant quelque temps. Or une telle justice n'existait pas parmi les hommes; elle ne pouvait donc être ni cachée ni plus tard manifestée. Elle aurait pu être donnée, connmuniquée aux hommes, aux croyants, mais elle ne pouvait pas leur être manifestée. La justice de Dieu, au contraire, existait, naturellement,

IEph I, 7, Apoc I, 5. V, 9; I Pier I, 18s.; Hebr IX, 12ss.; Rom V, 9; I Jean I, 7 etc. 
de toute éternité. Seulement, elle était cachice jusqu'à ce qu'enfin elle a été inanifistice dans l'évangile.

$\mathrm{La}$ bikalocúvn $\Theta$ coû ne peut donc pas ètre une qualité communiquée aux hommes par Dieu. Partout et toujours dans l'Ancien et dans le Noveau T., c'est un attribut de Dieu. Ce qui le prouve, outre l'usage constant de cette locution, c'est qu'elle est ici en antithèse avec la colère de Dieu. Avant la venue de J.-C. c'était la colère de Dieu qui se' manifestait du ciel contre les injustices des hommes (I, I8), et par conséquent sa justice était cachée; mais maintenant dans l'évangile ce qui a été manifesté, ce qui se manifeste encore, c'est la'justice de Dieu (I, I7. III, 21, 25 et 26) et non plus sa colère. Dans ces 4 textes il est de

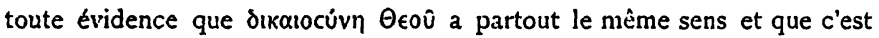
un attribut divin, qui est le contraire de lu colere.

Mais quand on sait que dans l'AT. la justice de Dieu est constamment mise en parallèle avec son salut, sa miséricorde etc., il n'y a pas lieu de s'en étonner; au contraire!

Ps XCVIII, 2 et 3: 'Ervẃplce Kúploc tò cwtíplov aủioû, Evavtíov

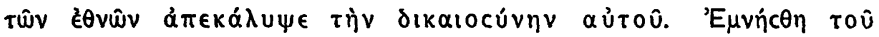

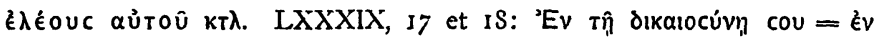

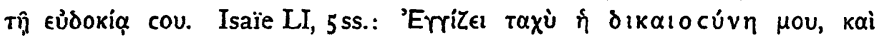

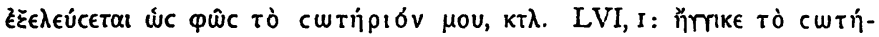

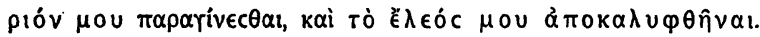

On voit le rapport étroit qui existe entre ces passages et ceux de l'épître aux Romains. Cf. aussi Ps CIII, 17. XXIV, 5. XXXVI, 7. I . XL, 11. LXXI, 2, 15 ss. etc. etc. Isaie XLV, 8, 19. XLVI, 13. LIX, I7 etc.

C'est au point que les LXX ont parfois traduit par oıkalocúvn. l'hébreu 7on bonté, , סחר compassion etc. (Gen XIX, 19; EX XXXIV, 7; Dan IX, 9. I3).

Enfin, dans la Sapience du Siracide, nous voyons par le texte hébreu

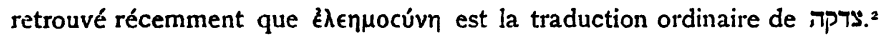

Il faut donc perdre l'habitude que nous avons d'identifier la justice de Dieu avec sa sévérité ou sa colère: dans le langage biblique elle en est précisément l'apbosé.

4. Avant de continuer le développement de sa pensée, l'apôtre revient en arrière, sur le temps passé: il réfléchit que l'ảmodútpweıc en J.-C. n'a pas eu seulernent pour but (eíc ou npóc) la manifestation de la

x V. Rerue de theologic de Montauban 1900, p. 299 ss. (La manifestation de la justice de Dieu dans l'évangile, par C. Bruston.)

2 Sir. III, 14, 30. VII, 10. XII, 3 etc. 
justice de Dicu dans le temps présent, mais qu'elle a aussi eu pour cause

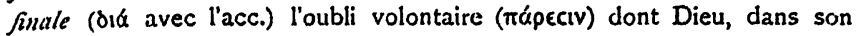
support, a usć à l'égard des péchés commis précédemment: dià tìv

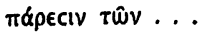

Entre eic et $\pi \rho \delta c$, oló ne peut indiquer qu'une cause finale ou un but, comme dans IV, 25 et ailleurs. Le parallelisme qui existe entre la

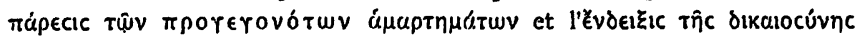

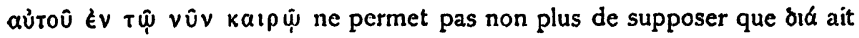
ici un sens tout different de celui de $\pi$ póc. Enfin le sens de cause efficiente ou antérieure ne clonnc aucune idée intelligible dans le contexte, car à supposer que la mápecıc fût antérieure à la manifestation de la justice de Dieu, il est clair qu'elle n'en est pas la cause. La seule cause en

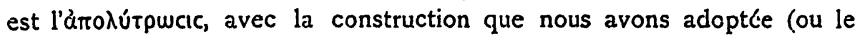
décret divin ou Jésus i $\lambda \alpha$ ctńpıov, avec la construction ordinaire).

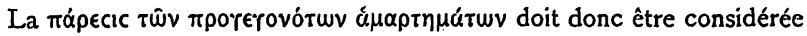

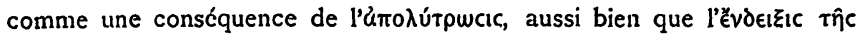
Jıkaıocúvnc aủroû. Il s'agit, non de ce que Dieu fit jadis, mais de ce qu'il a bien voulu faire récemment, en considération de l'åmohútpwcic ou de l'œuvre de J.-C.: „dans son support" ou sa bonté (cf. II, 4), il a consenti à considérer comme non avenus les péchés des temps passés, ou à úmepidê̂v toùc xpóvouc tîc árvoíac (Act XVII, 30), - ce qui signifie à peu près la même chose.

5. Après cela, l'apôtre reprend la phrase commencée, en y remplaçant seulement la préposition par une préposition synonyme: rpòc

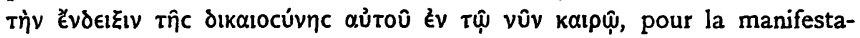
tion de sa justice (bonté) dans le temps présent (et à l'avenir, pour ceux

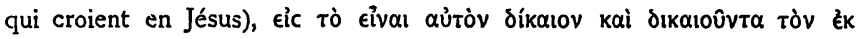
Tíctewc ['Incov̂].

Cette manifestation de sa justice pour le présent et l'avenir a pour but et pour résultat que Dieu est juste (!) .... C'est ainsi qu'on traduit ordinairement; mais quoi? Dieu n'était-il donc pas juste avant la venue de J.-C.? Qui oserait le soutenir? La rédemption en Christ ne peut pas. avoir eu pour but de faire que Dieu soit ce qu'il était de toute éternité, ce qu'il est par nature et ne peut pas ne pas être.

Je considère díkalov comme une apposition à aủióv et je traduis: „pour que lui, (qui est) juste, soit aussi justifiant celui qui est de la foi [en Jésus]? $x$

I V. Revuse de théol. de Montauban, Igoo (article cité).

11. 2. 1906 . 
Le but de la rédemption n'est pas que Dieu soit juste (cela est impossible), mais qu'il justifie (ou rende juste) aussi celui qui a la foi.

Et cela est assez naturel puisqu'il a voulu de tout temps que Jésus-Christ fût un ìactípiov par la foi.

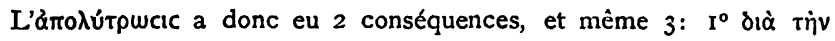

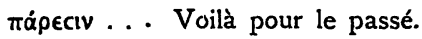

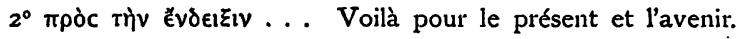

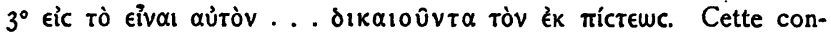
clusion de la phrase (ou plutôt de la protase) répond aussi bien que

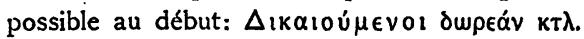

6. C'est qu'en effet ces 3 versets $(24-26)$ ne peuvent pas être la continuation de la phrase du v. 23: "Car tous ont péché, et sont privés de la gloire de Dieu", vu que si tous ont péché, tous ne sont pas justifiés gratuitement etc., mais seulement ceux qui croient.

Le professeur Oltranare, de Genève (1881), a fort bien vu qu'avec le v. 24 commence une nouvelle phrase, dont l'apodose ne se trouve qu'au v. 27:

„Etant justifiés gratuitement etc., où donc est la glorification? Elle a été exclue."

Tous les détails des v. $24-26$ montrent en effet que la justification est un don gratuit de Dieu par Christ et que par conséquent il n'y a pas lieu, pour ceux qui en sont l'objet, de se glorifier. Une telle tendance ou tentation a été exclue, non par la loi des œuvres, mais par celle de la foi, puisque (ráp) l'homme est justifié par la foi seule (v. 27-30).

Je termine ici ces quelques remarques, qui n'ont nullement pour but d'épuiser la matière (ce qui serait difficile), mais seulement de compléter et de rectifier sur quelques points l'étude si intéressante et si importante de M. Deissmann, en en tirant les conséquences logiques. 